\title{
Perioperative predictors of prolonged length of hospital stay following total knee arthroplasty: a retrospective study from a single center in China
}

Xiaoxiao Song ${ }^{1 \dagger}$, Caiwei Xia ${ }^{2 \dagger}$, Qiangqiang Li ${ }^{3,4}$, Chen Yao ${ }^{3,4}$, Yao Yao ${ }^{1,4}$, Dongyang Chen ${ }^{1,4^{*}}$ and Qing Jiang ${ }^{1,4^{*}}$

\begin{abstract}
Background: Decreasing the length of hospital stay is an ideal course of action to appropriately allocate medical resources. The aim of this retrospective study was to identify perioperative factors that may decrease the length of hospital stay (LOS).

Methods: In this study, we collected the data on 1112 patients who underwent primary total knee arthroplasty surgery (TKAs) at our institution from Jan 1, 2011 to Nov 31, 2017. Based on the published literature, 16 potential factors ( 12 preoperative variables, 1 intraoperative variable, and 3 postoperative variables) were investigated. The patients requiring a hospital stay longer than the mean LOS ( 8 days) were defined as patients with a prolonged LOS. The factors with a $P$ value less than 0.1 in the univariate analysis were further analysed in a multivariate model. An ordinal regression was used to determine independent risk factors for a prolonged LOS.

Results: The mean LOS was 8.3 days ( \pm 4.3$)$, with a range of 2 to 30 days. Sixteen variables were analysed by univariate analysis, and 11 of them had $p<0.1$ and were included in the multivariable model. Finally, 9 factors were found to be associated with a prolonged LOS. Among the 9 variables, 2 were surgery-related factors (operative time and intraoperative blood loss), and 3 were patient-related factors (age, ASA classification and neurological comorbidities).

Conclusion: In this study, we found that the clinical protocol, complications, the patient's age, the ASA classification, neurological comorbidities, the operative time, the ward, intraoperative blood loss and the surgeon were all factors contributing to a prolonged LOS. In clinical practice, these factors provide important information for the surgeon and are useful for identifying patients with a high risk of a prolonged LOS.
\end{abstract}

Keywords: Length of hospital stay, Total knee arthroplasty, Operative time, Predictor

\section{Introduction}

Total knee arthroplasty (TKA) is a highly effective procedure for relieving pain and improving knee function for people suffering from advanced knee rheumatoid arthritis (RA) and osteoarthritis (OA) [1]. With the rise in healthcare costs, the demand for TKA surgery recently increased due to factors such as the ageing

\footnotetext{
*Correspondence: 28617621@qq.com; qingj@nju.edu.cn

'Xiaoxiao Song and Caiwei Xia contributed equally to this work.

'Department of Sports Medicine and Adult Reconstructive Surgery, Nanjing

Drum Tower Hospital, School of Medicine, Nanjing University, 321

Zhongshan Road, Nanjing 210008, Jiangsu, People's Republic of China

Full list of author information is available at the end of the article
}

population, improvement in the standard of living, pursuit of a high quality life, and the maturity of TKA surgery [2]. In addition, patients sometimes have to wait to be treated for days or even months and are not treated in a timely manner due to limited hospital resources [3]. To appropriate allocate resources, decreasing the length of hospital stay (LOS) is an ideal course of action [4].

In recent decades, there has been a considerable amount of progress in reducing the LOS [5] because many potential factors for prolonged LOS have been studied, including age, sex, comorbidities, weekend admission, the American Society of Anesthesiologists

(c) The Author(s). 2020 Open Access This article is distributed under the terms of the Creative Commons Attribution 4.0 International License (http://creativecommons.org/licenses/by/4.0/) which permits unrestricted use, distribution, and reproduction in any medium, provided you give appropriate credit to the original author(s) and the source, provide a link to the Creative Commons license, and indicate if changes were made. The Creative Commons Public Domain Dedication waiver (http://creativecommons.org/publicdomain/zero/1.0/) applies to the data made available in this article, unless otherwise stated. 
(ASA) classification, preoperative education, and the need for blood transfusion [6-13]. Additionally, with the introduction of fast-track protocols, which emphasize pain and blood management, limit perioperative complications, and promote postoperative early ambulation, the LOS has decreased dramatically without compromising functional recovery $[4,6]$.

However, fast-track protocols are not easy to implement in China for the following two reasons. First, as China is a developing country, extended care facilities such as skilled nursing facilities and rehabilitation units are not as prevalent in China as they are in developed countries [14]. Additionally, the roles of doctors and nurses in China are demanding; they do not have a sufficient amount of time to address discharged patients. Second, the relationship between doctors and patients is sometimes tense $[15,16]$, and patients often request a prolonged hospital stay to ensure that they can perform daily activities. This phenomenon is also common in other developing countries [7]; however, few studies have predicted the factors that influence LOS in this medical environment. Thus, we performed this study to determine which factors can significantly affect LOS after TKAs in this medical environment.

\section{Materials and methods}

All patients undergoing primary unilateral TKA in our hospital between Jan 1, 2011 and Dec 31, 2017 were enrolled in this study. Based on the previous literature, the following 16 potential factors were recorded: age, sex, clinical protocol, operative time, surgeon, postoperative complications, day of surgery, payment type, Barthel Index (BI) score, preoperative haemoglobin $(\mathrm{Hb})$, intraoperative blood loss, range of motion (ROM), transfusion, ASA classification, comorbidities and ward. All data were extracted by one author.

All TKAs were performed by four experienced orthopaedic surgeons. General anaesthesia and a tourniquet inflated to $300 \mathrm{mmHg}$ were used for all patients before the surgery started. As a routine practice, $100 \mathrm{ml}$ of cefazolin was administered intravenously approximately 30 min before the surgery. All TKAs were performed by the medial parapatellar approach. One drain was placed in the articular cavity after the surgery and was removed one to 2 days later. Postoperatively, a multimodal pain control medication, including $50 \mathrm{mg}$ of tramadol and a $50 \mathrm{mg}$ injection of flurbiprofen axetil or $40 \mathrm{mg}$ parecoxib sodium, was administered to all patients two times a day. Beginning in 2014, a new clinical protocol, which mainly included the following 3 processes, was performed: 1) building a special rehabilitation team aimed at helping patients regain function until they achieved the discharge criteria; 2) introducing a "cocktail pain control" model, including the injection of $10 \mathrm{mg}$ morphine and $150 \mathrm{mg}$ ropivacaine around the incision after the wound was closed; and 3) implementing sound perioperative management plan to prevent complications, which included the administration of $50 \mathrm{mg}$ of tranexamic acid intravenously to all patients after implantation to reduce blood loss and the routine administration of chemoprophylaxis (rivaroxaban or low molecular weight heparin) and mechanical prophylaxis (pneumatic compression with a foot pump) to all patients to prevent deep vein thrombosis (DVT).

Patients were permitted to be discharged after they met the following criteria: (1) safe and independent walking with a walking aid; (2) flexion angle of the knee $\geq 90^{\circ}$; (3) no complications or controlled complications; (4) adequate pain control with a VAS score of $<3$ at rest and $<5$ during mobilization; and (5) strongly demands to be discharged. Our therapists evaluated whether a patient had met the discharge criteria, discussed this evaluation with the surgeon, and finally, both of them determined whether the patient should be discharged.

All LOS values mentioned in this article correspond to the duration from the day of surgery to the day of discharge $[13,17]$. The patients requiring a hospital stay longer than the mean LOS (8 days) were regarded as patients with a prolonged LOS. In this study, we collected data from the medical database, and patients' private information was not retrieved. Therefore, consent for participation was waived.

\section{Statistical analysis}

Statistical analyses were performed with SPSS version 23.0 (SPSS Inc., Chicago, IL, USA). Firstly, we performed exploitative analysis to detect the collinearity and avoid obvious internal correlation among the 16 variables. Then, in the univariate analysis, we used one-way ANOVA to describe the associations between continuous variables and LOS and the chi-squared test to describe the associations between categorical variables and LOS. The risk factors with a $p$ value less than 0.1 in the univariate analysis were used in the multivariate analysis. In the multivariate analysis, we used binary regression to determine the independent risk factors for increased LOS. Odds ratios and 95\% confidence intervals (CIs) were reported. A p value less than 0.05 was used to determine statistical significance.

\section{Results}

In the exploitative analysis, no collinearity was found. The demographic data are presented in Table 1. A total of 1112 patients were included in this study. There were $927(83.5 \%)$ females and $183(16.5 \%)$ males. The mean age was 67.9 years, and the age range was from 28 to 89 years. The mean LOS for all patients was $8.3 \pm 4.3$ days and ranged from 2 to 30 days. 
Table 1 Demographic features of 1112 patients who underwent total knee arthroplasty surgery

\begin{tabular}{|c|c|c|c|c|}
\hline Variables & Sample, N (\%) & Mean \pm SD & Mean LOS (days) & $P$-value \\
\hline Age (years) & & $67.9 \pm 7.9$ & & 0.036 \\
\hline$\leq 65$ & $396(35.7)$ & & $7.9 \pm 2.4$ & \\
\hline$>65$ & $714(64.3)$ & & $9.5 \pm 3.6$ & \\
\hline Gender & & & & 0.441 \\
\hline Female & $927(83.5)$ & & $8.3 \pm 4.3$ & \\
\hline Male & $183(16.5)$ & & $8.0 \pm 4.2$ & \\
\hline Clinical protocol & & & & $<0.01$ \\
\hline Traditional protocol & $71(6.4)$ & & $11.6 \pm 2.3$ & \\
\hline Current protocol & $294(26.5)$ & & $7.2 \pm 1.9$ & \\
\hline Operative time(min) & & $89.3 \pm 21.5$ & & $<0.01$ \\
\hline$\leq 70$ & $253(22.8)$ & & $7.4 \pm 4.0$ & \\
\hline $70-110$ & $646(58.3)$ & & $8.2 \pm 4.3$ & \\
\hline$\geq 110$ & $210(18.9)$ & & $9.5 \pm 4.2$ & \\
\hline Surgeon & & & & $<0.01$ \\
\hline 1 & $297(26.8)$ & & $7.9 \pm 4.5$ & \\
\hline 2 & $320(28.9)$ & & $8.57 \pm 4.1$ & \\
\hline 3 & $306(27.6)$ & & $7.4 \pm 4.0$ & \\
\hline 4 & $186(16.8)$ & & $10.2 \pm 3.7$ & \\
\hline $\mathrm{ROM}$ & & $91.3 \pm 18.0$ & & $<0.01$ \\
\hline$<90^{\circ}$ & $344(35.4)$ & & $8.6 \pm 4.4$ & \\
\hline$\geq 90^{\circ}$ & $583(64.6)$ & & $7.7 \pm 4.1$ & \\
\hline Complications & & & & $<0.01$ \\
\hline Yes & $220(19.8)$ & & $11.0 \pm 4.2$ & \\
\hline No & $892(80.2)$ & & $7.6 \pm 4.0$ & \\
\hline Intraoperative blood loss(ml) & & $219.8 \pm 120.5$ & & $<0.01$ \\
\hline$\leq 150$ & $291(41.3)$ & & $7.7 \pm 4.0$ & \\
\hline $150-300$ & $287(40.8)$ & & $8.2 \pm 4.2$ & \\
\hline$\geq 300$ & $126(17.9)$ & & $10.6 \pm 4.0$ & \\
\hline Preoperative $\mathrm{Hb}(\mathrm{g} / \mathrm{L})$ & & $127.9 \pm 13.7$ & & 0.571 \\
\hline$\leq 110$ & $108(9.7)$ & & $7.6 \pm 4.4$ & \\
\hline $110-140$ & $786(70.8)$ & & $8.5 \pm 4.3$ & \\
\hline$\geq 140$ & $116(10.5)$ & & $7.3 \pm 4.1$ & \\
\hline BI Score & & & & 0.512 \\
\hline$\leq 90$ & $576(67.6)$ & & $7.3 \pm 4.0$ & \\
\hline$>90$ & $276(32.4)$ & & $7.8 \pm 4.2$ & \\
\hline Day of operation & & & & 0.221 \\
\hline Monday & $245(22.0)$ & & $8.3 \pm 4.4$ & \\
\hline Tuesday & $236(21.2)$ & & $8.9 \pm 4.1$ & \\
\hline Wednesday & $168(15.1)$ & & $7.6 \pm 3.9$ & \\
\hline Thursday & $212(19.1)$ & & $8.4 \pm 4.4$ & \\
\hline Friday & $210(18.9)$ & & $8.1 \pm 4.0$ & \\
\hline Type of pay & & & & 0.412 \\
\hline Selfpay & $577(60.8)$ & & $8.0 \pm 4.1$ & \\
\hline Medicare & $372(39.2)$ & & $8.2 \pm 4.4$ & \\
\hline
\end{tabular}


Table 1 Demographic features of 1112 patients who underwent total knee arthroplasty surgery (Continued)

\begin{tabular}{|c|c|c|c|c|}
\hline Variables & Sample, N (\%) & Mean \pm SD & Mean LOS (days) & $P$-value \\
\hline Ward & & & & $<0.01$ \\
\hline A & $923(83.2)$ & & $8.0 \pm 4.2$ & \\
\hline B & $186(16.8)$ & & $9.8 \pm 4.1$ & \\
\hline Transfusion & & & & $<0.01$ \\
\hline Yes & $87(7.8)$ & & $11.0 \pm 5.2$ & \\
\hline No & 1025 (92.2) & & $8.1 \pm 4.1$ & \\
\hline ASA classification & & & & 0.015 \\
\hline$|/| \mid$ & $920(82.7)$ & & $8.1 \pm 3.5$ & \\
\hline III/IV & 192(17.3) & & $9.3 \pm 4.1$ & \\
\hline Comorbidities & & & & 0.043 \\
\hline Healthy & 674 & & $7.9 \pm 4$ & \\
\hline Cardiovascular & 85 & & $8.5 \pm 3.7$ & \\
\hline Neurological & 93 & & $9.7 \pm 3.2$ & \\
\hline
\end{tabular}

$P<0.05$ was considered statistically significant. ROM Range of motion, $B /$ Barthel Index. SD Standard deviation, $\mathrm{Hb}$ Hemoglobin

In this study, 220 (18.3\%) cases of complications were recorded, which mainly included infection, wound exudate, persistent fever and thrombogenesis. Patients with complications stayed in the hospital longer (11.6 days) than patients without complications (7.5 days), as depicted in Fig. 1. Patients in ward B stayed longer (9.8 days) than patients in ward A (8.0 days), as depicted in Fig. 2. Older patients stayed in the hospital longer $(>65$ years, 9.5 days) than the younger group ( $\leq 65$ years, 7.9 days, Fig. 3). Patients with ASA III/IV tended to stay longer (9.3 days) than those with ASA I/II (8.1 days, Fig. 4). Patients with neurological disease stayed in the hospital longer (9.7 days) than healthy patients (7.9 days, Fig. 5), and patients undergoing surgery with the traditional protocol stayed in the hospital longer than those undergoing surgery with the current protocol, as depicted in Fig. 6. A downward trend in the LOS was noted with a decrease in intraoperative blood loss, as depicted in Fig. 7. Similarly, for every increase in

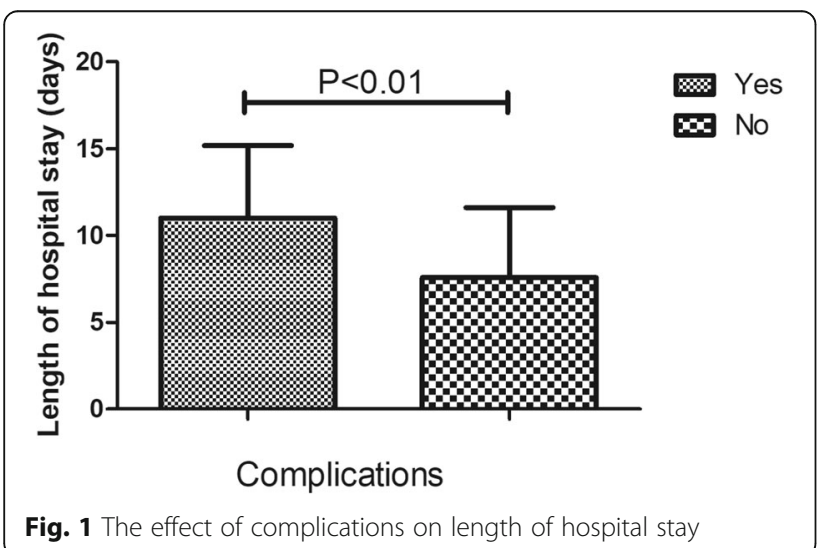

operative time by $30 \mathrm{~min}$, the LOS increased by 10.8 to $15.9 \%$, as depicted in Fig. 8. The selection of the surgeon can also influence LOS, as depicted in Fig. 9.

In the univariate analysis, the patient's age, the ward, the clinical protocol, the operative time, the surgeon, complications, intraoperative blood loss, transfusion, intraoperative blood loss, and ROM showed an association with a prolonged LOS (Table 1). After adjusting for other variables, 9 factors were found to be associated with prolonged length of stay, including complications $(p<0.01)$, the patient's age $(p=0.032)$, the clinical protocol $(p<0.01)$, intraoperative blood loss $(p=0.029)$, the operative time $(p=0.048)$, the surgeon $(p=0.015)$, the ward $(p<0.01)$, the ASA classification $(p=0.045)$, and comorbidities $(p=0.027)$ (Table 2).

\section{Discussion}

In this study, we investigated 16 potential factors and found that 9 factors were associated with a prolonged

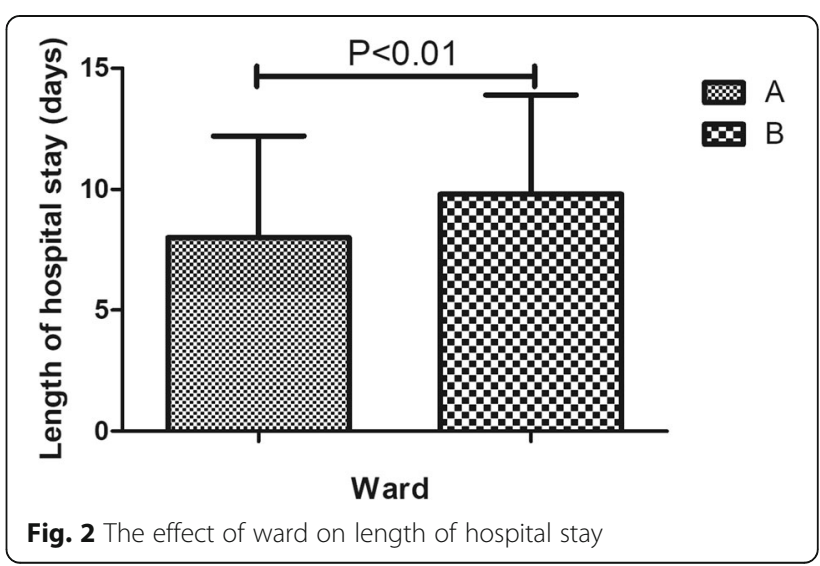




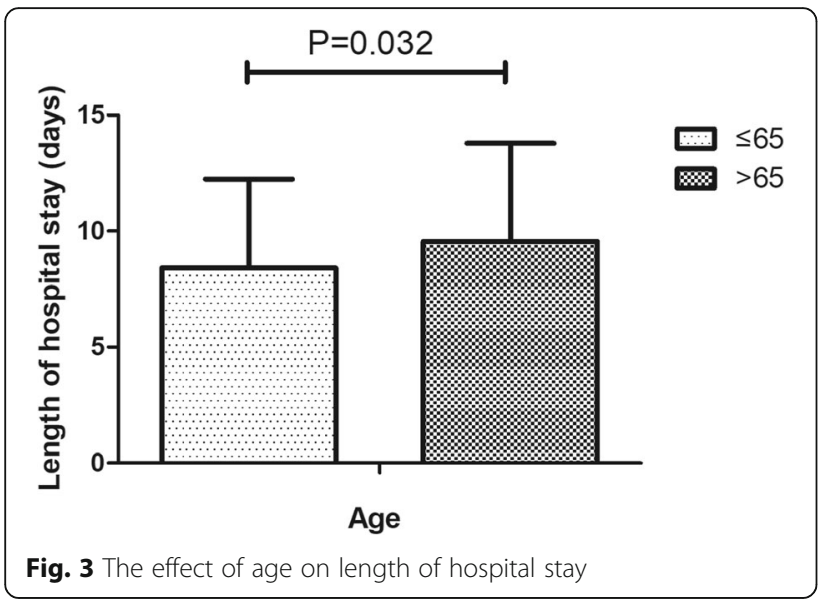

LOS, including postoperative complications, the patient's age, the clinical protocol, intraoperative blood loss, the operative time, the surgeon, the ward, the ASA classification, and neurological comorbidities.

Complications were also found to be a contributing factor for prolonged LOS in previous studies [7, 8]. Patients in our centre were not allowed to be discharged from the hospital once complications such as DVT and infection occurred unless the complications were controlled.

Patients in ward A tended to have shorter hospital stays than those in ward B, which is in accordance with the findings in a previous study [13]. In our department, ward A had more trained therapists and surgeons, which partly explains the faster turnover of patients in ward A.

Consistent with the results of other studies, the older group of patients ( $>65$ years) was likely to stay in the hospital longer than the younger group ( $\leq 65$ years) [ 7 , 18-20]. Older patients tend to be in worse physical condition and more likely to have postoperative complications [21, 22], which increases the LOS. Furthermore, older patients are more likely to live alone and need caregiver support than are younger patients, and these factors have been shown to be important predictive factors for an extended LOS [6].

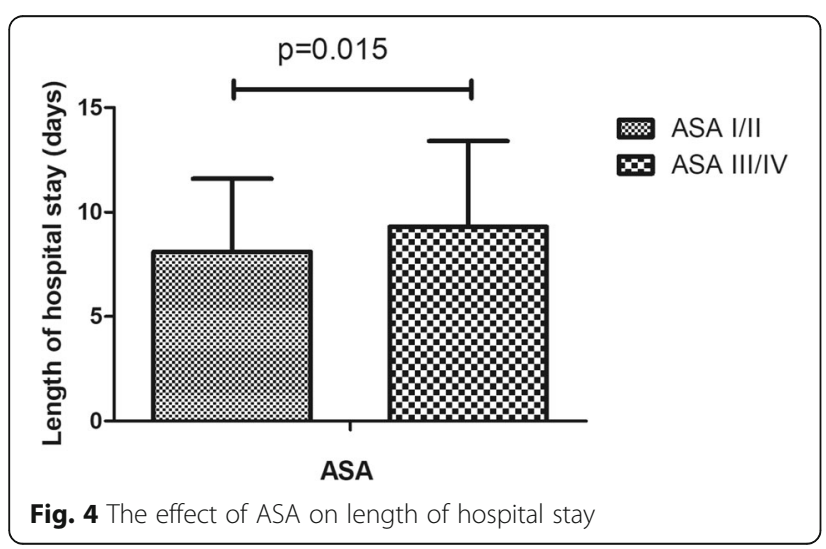

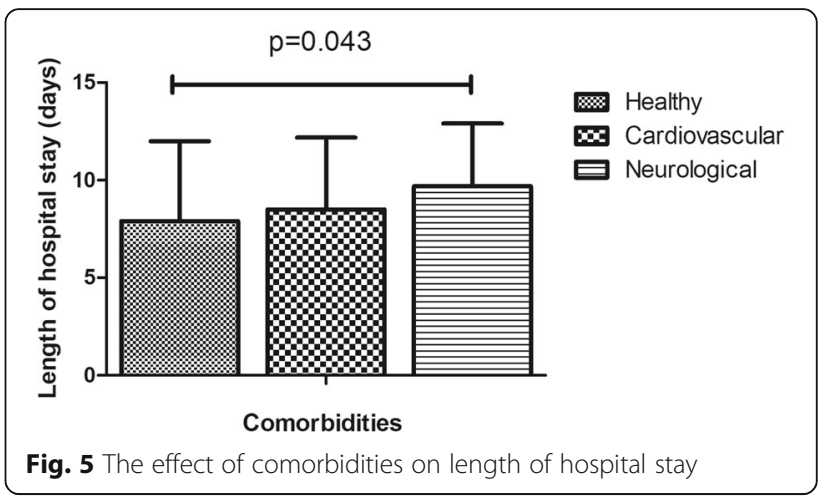

The ASA classification is frequently used to define patients' physical condition [11, 13]. It is reasonable to conclude that patients with ASA III/IV often stay longer than those with ASA I/II, as they require much more time to reach the strict discharge criteria. Additionally, we assessed the influence of comorbidities and found that neurological comorbidities can prolong hospital stays. We speculate that neurological diseases make it difficult for patients to exercise soon after surgery. In contrast to the results of other literature, we did not find a statistically significant association between cardiopulmonary disease and prolonged LOS, which may be due to the small sample sizes.

Beginning in 2014, a new clinical protocol was implemented in our department. Comparing with the traditional clinical protocol, the new one is more reasonable and sound owning to the improvement of some perioperative management protocol, including building a new rehabilitation team and introducing a "cocktail pain control" model. In this study, we investigated whether a good perioperative protocol can change patients' LOS. Our results showed that people stayed in the hospital for a much shorter period when the protocol was implemented in patients who received TKA surgery. Thus, hospitals in developing countries should develop sound perioperative management plans to shorten LOS and increase the medical resource utilization rate.

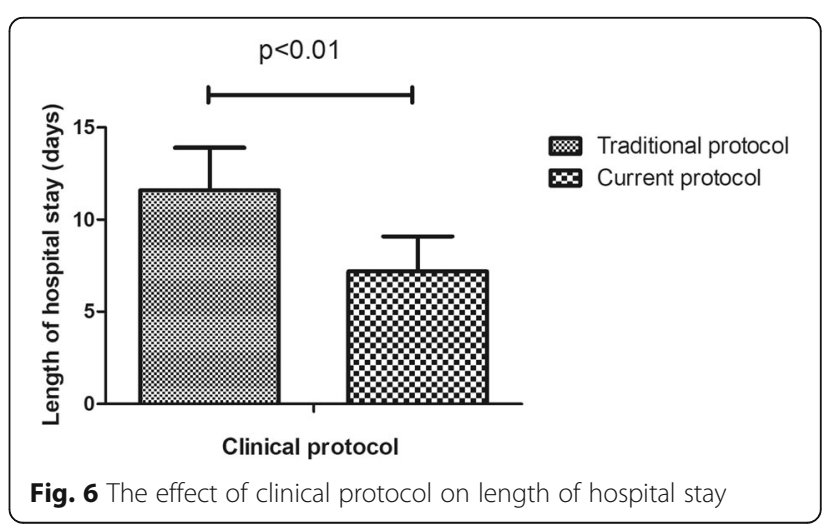




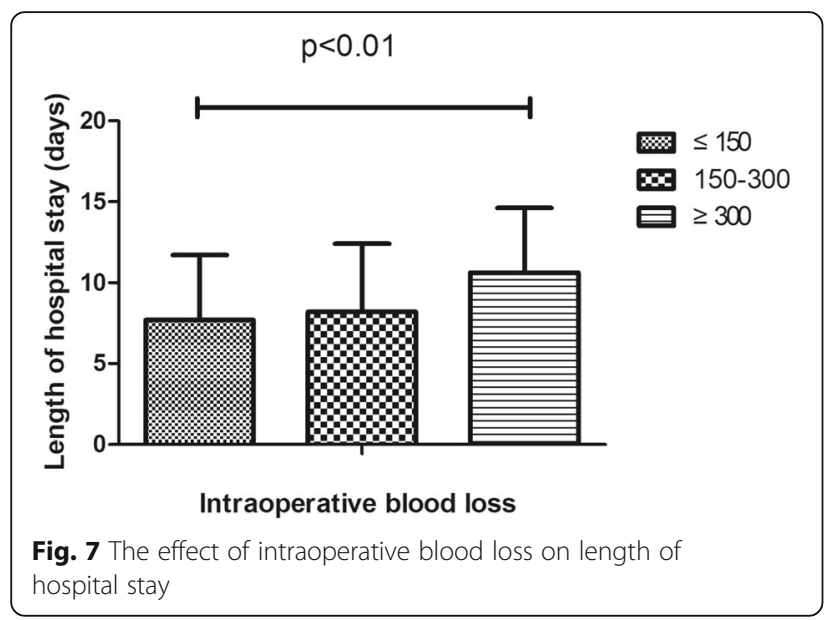

We found that a longer operative time is positively correlated with a prolonged LOS, which is in accordance with the results of a study by Ifeoma et al. [11]. An explanation for this result might be that a tourniquet is routinely used for patients undergoing TKAs in our institution. Therefore, when the operative time increased, the tourniquet time increased accordingly. The hypoxiaischaemia induced by the use of tourniquet may result in the swelling of soft tissue, weakened strength of the quadriceps muscle and pain in the thigh [23, 24], which slows early rehabilitation training and consequently leads to a prolonged LOS; another explanation may be the positive correlation between a prolonged operative time and an increased infection rate after TKAs [25].

We found that increased intraoperative blood loss is associated with a prolonged LOS, which has not been previously reported. A possible reason for this correlation may be that the loss of blood during an operation can make patients feel weak and make it difficult for them to perform early physical exercise.

In this study, we found that surgeons have a positive effect on LOS following TKA, which is consistent with the finding of Monsef et al. [17]. A possible explanation for

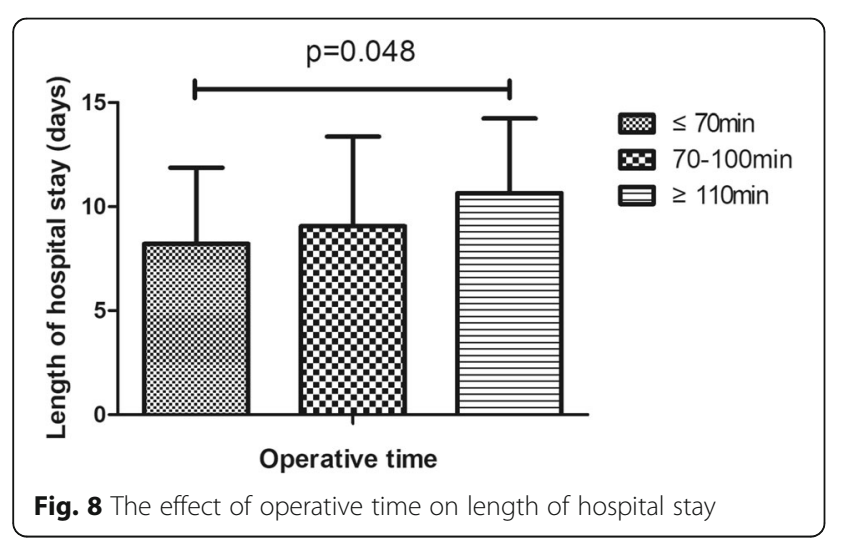

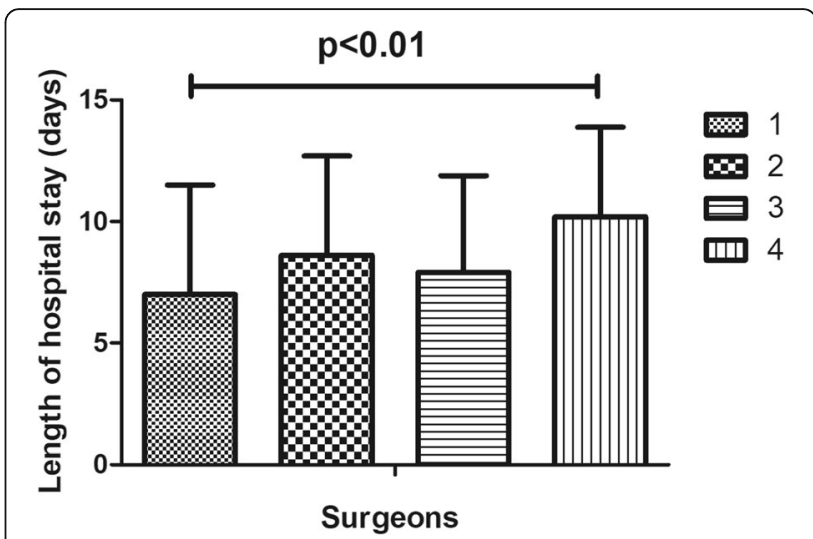

Fig. 9 The effect of surgeon on length of hospital stay

this result may be that surgeons had different experiences in managing patients during the perioperative period. Additionally, they may have been relaxed or firm when determining whether the patients should be discharged.

In reality, patients' decisions may affect LOS because they have the right to decide when to leave hospital. However, it

Table 2 Binary regression model for perioperative predictors of prolonged length of hospital stay

\begin{tabular}{lll}
\hline Variables & $P$-value & Odds Ratio $(95 \% \mathrm{Cl})$ \\
\hline $\begin{array}{l}\text { Age }(>65 \text { vs } \leq 65) \\
\text { Clinical protocol (vs current) }\end{array}$ & 0.032 & $1.98(0.85$ to 1.41$)$ \\
$\quad$ Traditional & $<0.01$ & $6.81(3.85$ to 12.04$)$ \\
$\begin{array}{l}\text { Operative time }(\geq 110 \text { min vs) } \\
\leq 70 \text { min }\end{array}$ & 0.048 & $1.96(0.99$ to 3.89$)$ \\
$70-110$ min & ns & $1.19(0.86$ to 2.69$)$ \\
Surgeon (vs 4) & & \\
1 & 0.015 & $0.39(0.03$ to 0.30$)$ \\
2 & ns & $0.77(0.42$ to 1.43$)$ \\
3 & ns & $0.86(0.47$ to 1.40$)$
\end{tabular}

Comorbidities (vs healty)

\begin{tabular}{|c|c|c|}
\hline Cardiovascular & ns & 1.14 (0.56 to 1.78$)$ \\
\hline Neurological & 0.027 & 2.13 (2.11 to 3.32$)$ \\
\hline ASA classification (III/IV vs I/II) & 0.045 & 1.91 (1.01 to 3.59$)$ \\
\hline $\mathrm{ROM}\left(<90^{\circ} \mathrm{vs} \geq 90^{\circ}\right)$ & ns & $1.31(-0.14$ to 0.49$)$ \\
\hline Transfusion (no vs yes) & ns & $0.82(0.53$ to 1,23$)$ \\
\hline Complications (no vs yes) & $<0.01$ & 0.28 (0.14 to 0.56$)$ \\
\hline Ward (B vs A) & $<0.01$ & $4.23(1.76$ to 3.14$)$ \\
\hline \multicolumn{3}{|c|}{ ntraoperative blood loss( $\geq 300 \mathrm{ml} v \mathrm{vs})$} \\
\hline$\leq 150$ & 0.029 & 2.05 (1.08 to 3.92$)$ \\
\hline $150-300$ & ns & 1.71 (0.97 to 3.40$)$ \\
\hline Preoperative $\mathrm{Hb}(\mathrm{g} / \mathrm{L})$ & ns & $0.98(0.32$ to 1.41$)$ \\
\hline
\end{tabular}

$P<0.05$ was considered statistically significant. $R O M$ Range of motion, $\mathrm{Cl}$ Confidence interval 
is difficult for us to record patients' preferences and analyse these data. The patients who want stay in the hospital for a longer period may have common traits, such as being discharged to their home, living alone and having no caregiver support, which have been suggested as predictors of a prolonged LOS [26]. Unfortunately, in our database, this information was not recorded. We hope that these patientrelated variables will be analysed in the future.

There is no consensus on the contribution of sex to a prolonged LOS. Some researchers have found that females tend to have a longer LOS than males $[6,11,27]$. The authors explained that females are more likely to have higher rates of obesity, postoperative transfusion and postoperative complications. However, some studies did not find statistically significant differences in LOS between the two groups $[10,12]$. Similarly, in our study, the difference between males and females was not obvious. However, this result may be caused by the unbalanced sex ratio (1:3). Therefore, more studies should be performed to investigate the effect of sex on LOS. In addition to sex, other variables such as the BI score, the VAS score, ROM, the day of operation, and transfusion were not associated with prolonged LOS in our study.

There are some limitations to this study. First, we split continuous variables into groups based on the sample mean, which is the most common approach used in the literature. Information loss is inevitable, and therefore, the statistical power to detect a correlation may have been weakened. Second, we extracted all data from medical records, and the data for several factors, such as the VAS score, ROM and intraoperative blood loss, may not be accurate; however, bias can be accommodated due to the large sample size. Third, all of the patients analysed were from our institution; thus, the result may not be representative of all hospitals in China.

Despite the above limitations, this study has important clinical value for several reasons. First, this is the first article to explore the association between perioperative variables and LOS following TKAs in China. Moreover, we found nine factors that positively contribute to a prolonged LOS. This result is reliable and meaningful because of the high quality of our centre compared with other orthopaedic clinics in China and the large sample size in this study, even though this is a single-centre study. In addition, all of the data in our study were collected from medical records rather than from a database. Therefore, we could investigate some variables that cannot be retrieved via procedural codes. Finally, 16 variables were included in the present study, which is much more than the number of variables included in other similar studies. Importantly, the sample size was sufficiently large to generate a reliable statistical outcome. Although fast-track protocols are prevalent in developed countries, the long LOS model is common among developing countries $[7,8]$. Therefore, it is essential to explore factors that can potentially reduce the LOS following TKAs in developing countries.

\section{Conclusion}

We found that complications, the patient's age, the clinical protocol, intraoperative blood loss, the operative time, the surgeon, the ward, the ASA classification, and neurological comorbidities are nine predictive factors contributing to a prolonged LOS. These factors can be used to provide counselling to patients and to further optimize perioperative protocols for the patients at risk for an increased LOS following TKAs.

\section{Abbreviations}

ASA: American Society of Anesthesiologists; DVT: Deep vein thrombosis; LOS: Length of hospital stay; OA: Osteoarthritis; RA: Rheumatoid arthritis; ROM: Range of motion; TKA: Total knee arthroplasty

\section{Acknowledgements}

None.

\section{Authors' contributions}

XXS participated in the design of the study, wrote the manuscript and performed the study, YY and CWX and QQL collected and analyzed the data. DYC and QJ designed and supervised the entire study. XXS was a major contributor in writing the manuscript. All authors read and approved the final manuscript.

\section{Funding}

This work was supported by the Projects of International Cooperation and Exchanges NSFC (81420108021), National Key Technology Support Program (2015BAI08B02), Excellent Young Scholars NSFC (81622033), Jiangsu

Provincial Key Medical Center Foundation, Jiangsu Provincial Medical Talent Foundation and Jiangsu Provincial Medical Outstanding Talent Foundation. The funding bodies played no role in the design of the study and collection, analysis, and interpretation of data and in writing the manuscript.

\section{Availability of data and materials}

The datasets used and/or analyzed during the current study will be available from author XXS on a reasonable request.

\section{Ethics approval and consent to participate}

No patients' private information is involved. Therefore ethics approval is not needed from our Research Ethics Committee and consent for participation was waived. The corresponding author (QJ) who is head of our department granted permission to access the raw data.

\section{Consent for publication}

Not applicable.

\section{Competing interests}

The authors declare that they have no competing interests.

\section{Author details}

'Department of Sports Medicine and Adult Reconstructive Surgery, Nanjing Drum Tower Hospital, School of Medicine, Nanjing University, 321 Zhongshan Road, Nanjing 210008, Jiangsu, People's Republic of China.

Department of Orthopedics, Taikang Xianlin Drum Tower Hospital, Nanjing University School of Medicine, Nanjing, Jiangsu, People's Republic of China. ${ }^{3}$ Department of Sports Medicine and Adult Reconstructive Surgery, Nanjing Drum Tower Hospital, Clinical College of Nanjing Medical University, Nanjing, China. ${ }^{4}$ Laboratory for Bone and Joint Diseases, Model Animal Research Center, Nanjing University, Nanjing 210093, Jiangsu, People's Republic of China. 
Received: 23 January 2018 Accepted: 6 January 2020

Published online: 31 January 2020

\section{References}

1. Jones CA, Beaupre LA, Johnston DW, Suarez-Almazor ME. Total joint arthroplasties: current concepts of patient outcomes after surgery. Rheum Dis Clin N Am. 2007;33(1):71-86.

2. Kurtz S, Ong K, Lau E, Mowat F, Halpern M. Projections of primary and revision hip and knee arthroplasty in the United States from 2005 to 2030. J Bone Joint Surg Am. 2007;89(4):780-5

3. Jain NB, Higgins LD, Ozumba D, Guller U, Cronin M, Pietrobon R, Katz JN. Trends in epidemiology of knee arthroplasty in the United States, 19902000. Arthritis Rheum. 2005:52(12):3928-33.

4. Specht K, Kjaersgaard-Andersen P, Pedersen BD. Patient experience in fasttrack hip and knee arthroplasty--a qualitative study. J Clin Nurs. 2016:25(5-6):836-45.

5. Kehlet $\mathrm{H}$, Thienpont E. Fast-track knee arthroplasty -- status and future challenges. Knee. 2013;20(Suppl 1):S29-33.

6. Mathijssen NM, Verburg H, van Leeuwen CC, Molenaar TL, Hannink G. Factors influencing length of hospital stay after primary total knee arthroplasty in a fast-track setting. Knee Surg Sports Traumatol Arthrosc. 2016:24(8):2692-6.

7. Maiorano E, Bodini BD, Cavaiani F, Pelosi C, Sansone V. Length of stay and short-term functional outcomes after total knee arthroplasty: can we predict them? Knee. 2017;24(1):116-20.

8. El Bitar YF, Illingworth KD, Scaife SL, Horberg JV, Saleh KJ. Hospital length of stay following primary Total knee Arthroplasty: data from the Nationwide inpatient sample database. J Arthroplast. 2015;30(10):1710-5.

9. Pugely AJ, Martin CT, Gao Y, Belatti DA, Callaghan JJ. Comorbidities in patients undergoing total knee arthroplasty: do they influence hospital costs and length of stay? Clin Orthop Relat Res. 2014;472(12):3943-50.

10. Newman JM, Szubski CR, Barsoum WK, Higuera CA, Molloy RM, Murray TG. Day of surgery affects length of stay and charges in primary Total hip and knee Arthroplasty. J Arthroplast. 2017;32(1):11-5.

11. Inneh IA. The combined influence of Sociodemographic, preoperative comorbid and intraoperative factors on longer length of stay after elective primary Total knee Arthroplasty. J Arthroplast. 2015:30(11):1883-6.

12. Halawi MJ, Vovos TJ, Green CL, Wellman SS, Attarian DE, Bolognesi MP. Preoperative predictors of extended hospital length of stay following total knee arthroplasty. J Arthroplast. 2015;30(3):361-4.

13. van den Belt $L$, van Essen P, Heesterbeek PJ, Defoort KC. Predictive factors of length of hospital stay after primary total knee arthroplasty. Knee Surg Sports Traumatol Arthrosc. 2015;23(6):1856-62

14. Zhang Y, Zhang H, Clarke HD, Hattrup SJ. Analysis of total joint arthroplasty costs in Chinese patients. J Arthroplast. 2012;27(8):1423-8 e1421.

15. Hou X, Xiao L. An analysis of the changing doctor-patient relationship in China. J Int Bioethique. 2012;23(2):83-94 177-178.

16. He AJ. The doctor-patient relationship, defensive medicine and overprescription in Chinese public hospitals: evidence from a cross-sectional survey in Shenzhen city. Soc Sci Med. 2014;123:64-71.

17. Monsef JB, Della Valle AG, Mayman DJ, Marx RG, Ranawat AS, Boettner F. The impact of blood management on length of stay after primary total knee arthroplasty. Open Orthop J. 2014;8:108-13.

18. Ong PH, Pua $\mathrm{YH}$. A prediction model for length of stay after total and unicompartmental knee replacement. Bone Joint J. 2013;95-B(11):1490-6.

19. Jonas SC, Smith HK, Blair PS, Dacombe P, Weale AE. Factors influencing length of stay following primary total knee replacement in a UK specialist orthopaedic Centre. Knee. 2013;20(5):310-5.

20. Tan C, Loo G, Pua YH, Chong HC, Yeo W, Ong PH, Lo NN, Allison G. Predicting discharge outcomes after total knee replacement using the risk assessment and predictor tool. Physiotherapy. 2014;100(2):176-81.

21. Fang $M$, Noiseux $N$, Linson $E$, Cram $P$. The effect of advancing age on Total joint replacement outcomes. Geriatr Orthop Surg Rehabil. 2015;6(3):173-9.

22. Kennedy JW, Johnston L, Cochrane L, Boscainos PJ. Total knee arthroplasty in the elderly: does age affect pain, function or complications? Clin Orthop Relat Res. 2013:471(6):1964-9.

23. Liu D, Graham D, Gillies K, Gillies RM. Effects of tourniquet use on quadriceps function and pain in total knee arthroplasty. Knee Surg Relat Res. 2014;26(4):207-13.

24. Guler O, Mahirogullari M, Isyar M, Piskin A, Yalcin S, Mutlu S, Sahin B. Comparison of quadriceps muscle volume after unilateral total knee arthroplasty with and without tourniquet use. Knee Surg Sports Traumatol Arthrosc. 2016;24(8):2595-605.

25. Peersman G, Laskin R, Davis J, Peterson MG, Richart T. Prolonged operative time correlates with increased infection rate after total knee arthroplasty. HSS J. 2006:2(1):70-2.

26. Halawi MJ, Vovos TJ, Bolognesi MP, Green CL. Patient expectation is the Most important predictor of discharge destination after primary Total joint Arthroplasty. J Arthroplast. 2015;30(15):539-42.

27. Whitlock KG, Piponov HI, Shah SH, Wang OJ, Gonzalez MH. Gender role in Total knee Arthroplasty: a retrospective analysis of perioperative outcomes in US patients. J Arthroplast. 2016;31(12):2736-40.

\section{Publisher's Note}

Springer Nature remains neutral with regard to jurisdictional claims in published maps and institutional affiliations.
Ready to submit your research? Choose BMC and benefit from:

- fast, convenient online submission

- thorough peer review by experienced researchers in your field

- rapid publication on acceptance

- support for research data, including large and complex data types

- gold Open Access which fosters wider collaboration and increased citations

- maximum visibility for your research: over $100 \mathrm{M}$ website views per year

At $\mathrm{BMC}$, research is always in progress.

Learn more biomedcentral.com/submissions 